\title{
Cognition and asynchronous distribution between human and machine building accidents
}

\author{
Edgard Martins ${ }^{1 *}$, Marcelo Soares ${ }^{2}$, Lia Augusto $^{3}$, Laura Laura ${ }^{2}$ \\ ${ }^{1}$ Universidade Federal de Pernambuco- Depto de Design- CAA - Caruaru- Pernambuco- Brasil, \\ ${ }^{2}$ UFPE- Universidade Federal de Pernambuco- Depto de Design- CAA - Recife- Pernambuco- Brasil \\ ${ }^{3}$ FIOCRUZ, ${ }^{3}$ Centro de Pesquisas Aggeu Magalhães, Cidade Universitária,Recife, Pernambuco, Brasil
}

\begin{abstract}
The creation of meaning in communication is a trading activity, resulting from the construction that is born of the interaction between subjects. That is, the meaning is not inherent to the relationship between words, signs and symbols that arise from negotiating a necessary and unavoidable. As the concepts of sense as discrete and static representations imply a notion of classical computing and design of a cognitive system corresponding conceptions of meaning construction as located and shared among agents implies notions of different computing and cognition. Several efforts have been developed to meet these demands. Among them are the Connectionism (also known as neural networks. Records on aspects of mental health and stress of flight professionals are present in the official reports of the organs of investigation of aviation accidents worldwide since its inception. Problems related to health physical and mental health of pilots (fatigue, stress, physiological and psychosocial problems) account for $19 \%$ of causal factors in aircraft accidents. The training seems a paradox when we know that these professionals receive regular training, have high education and technical training of high level. However, problems arise related to the implementation of learning that can be influenced to reduce their cognitive capacity, making it in practice, relatively unable to exercise its functions effectively and safely.
\end{abstract}

Keywords: Distributed cognition, Automation, Behavior of mind

\section{Introduction}

Few people still believe that mind, thought and brain are separate things. We are what the brain generates, and we understand the world the way the brain can sense what is around us, especially according to the way we can extract information that surrounds us. The feelings, moods and emotions have effects on the central nervous system and the responses of the mechanisms governing the perception. The feelings, emotions and moods have a huge influence on memory.

The human mind encompasses much more than memory. Mental functions involved in perception, the alert level, the selection of what we perceive, remember or learn, the decision about what we do or not do, will, understanding, feelings, emotions, states ${ }^{*}$

\footnotetext{
"Corresponding author. Email: edgardpiloto@gmail.com
}

of spirit and all that is encompassed in the concepts of intelligence and consciousness.

All mental functions are strongly influenced by memories and vice versa, but are separate entities and with the same mechanisms themselves. Of course, all these phenomena in turn affect the short and longterm nerve activity and, within it, the mental functions, including those related to memory. There is a mind / body that is the basis of daily activity of both, and also called psychosomatic pathology, that not only exists as a cornerstone of modern medicine and psychiatry. The repeated stress can alter some of the physiological parameters (blood pressure, heart rate, gastric secretion) permanently. The mind is easy to describe in its broader aspects, but mental function in the specific circumstances of our lives remains a mystery. How to predict, before a given circumstance, if there is any remote intersection of information that will react in some unexpected way? 
Cognition can be conceived as processes that occur dynamically, in which time is essential (Port \& van Gelder 1995)[1]. Are central features of cognitive systems:

(a) be self-organized,

(b) and Adaptive

(c) display properties, settings or emerging structures.

The network infers, the story of his interaction with these examples, relevant features that change the internal settings of the network itself and allow you to, so adaptive, learning to perform a particular task. The final configuration of the network emerges from the learning process. A complex system composed of many entities interacting in a non-linear - presents an emergent property when it is caused by the interaction, according to the dynamics of the system.

\section{Distributed distributed cognition and emergency communication}

Distributed cognition can be understood as a distributed community of agents interacting with the environment in which this task is developed over time. The agents interact as a set of overlapping polygons constantly tensing and turning to each other. Figure 1, with a beehive metaphor makes this a dynamic process.

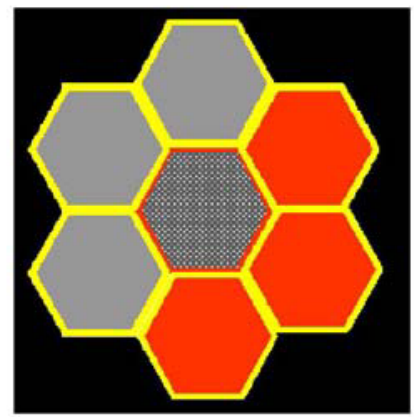

Figure 1 - In the hive is not "intent" of any bee to build hexagons Maximizing the area of the comb which is building the lead to try to build a circular shape.

Construction of a beehive. The final configuration of a network of knowledge emerges from the learning process. A complex system composed of many entities interacting in a non-linear - presents an emergent property when it is caused by the interaction, according to the dynamics of the system. Construction of a beehive. It is not "intent" of any bee to build hexagons (Figure 2)

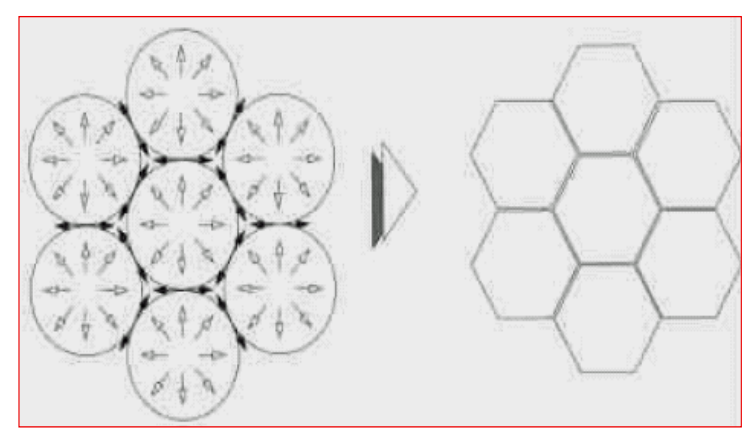

Figure 2 - In the hive is not "intent" of any bee to build hexagons. Maximizing the area of the comb which is building the lead to try to build a circular

The Connectionism (also known as neural networks are connections metaphorically in Figure 3, suggesting a constant dynamism between cognitive mental processes.

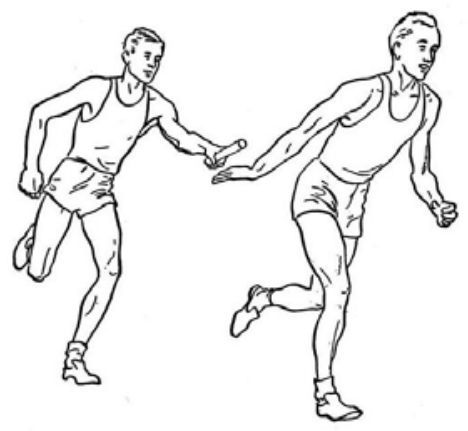

Figure 3 - The metaphor of the link between cognitive processes

\section{What happens}

What happens is that the work of each bee to maximize the area of the comb which is building the lead to try to br shape. However, around a circle can only put six other circles of the same size - in this case, other comb being built by other bees. Physical forces (surface tension) interact to deform the balls, causing them to take the form of hexagons. It is not "intent" of any bee to build hexagons. However, given the interaction between the autonomous actions of the bees and the restrictions imposed by the physical world, the hexagon is the only 
possible result. The hexagon emerges. It is also important to analyze the phenomenon at the highest level, that is, understanding the attribution of meaning as a joint activity and distributed. In traditional approaches the meaning lies in the mind of every cognitive agent. However, a design that gives preference to plastic and negotiated character of meaning, this can be understood as a distributed community of agents interacting with the environment in which this task is developed over time.

\section{4-Discussion}

A question may ask the command pilot of the aircraft highly automated: Who flies the plane: the human or flight instruments?

Scientists studying the interaction between humans and computers develop radically new ways of thinking about the interaction between pilots and the cockpit instruments. They claim that cognition is distributed among human beings that make up the cockpit and the flight team, the set of instruments to control the flight. By studying such situations cooperative scientists shed light on these features of cognition that are present in many other situations. Figure 4 enacts one representation workstation pilot where his task is divided with computers, establishing one scenario distributed cognition human / computer.

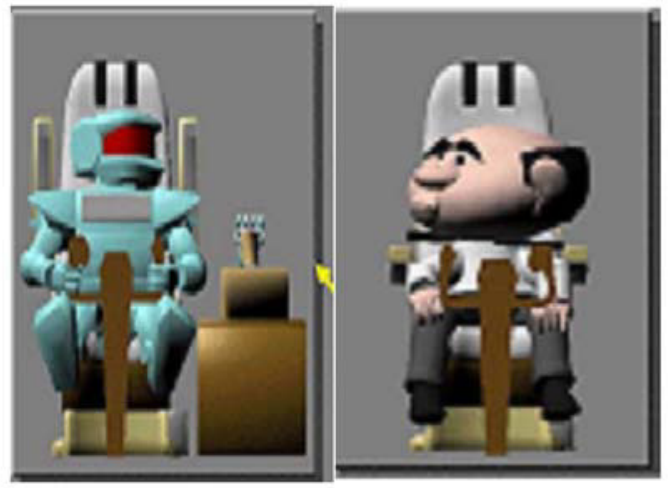

Figure 4 - The distribution of the task of steering between humans and computer

The landing of an aircraft depends on the correct configuration of the geometry of the wing and the speed of the plane. We need the speed of the aircraft is low enough to allow the landing safely. However, for certain configurations of the wing, a very low speed can mean the downfall of the device due to lack of support. Therefore, an essential task for the crew is to tailor the configuration of wings to the desired speed for landing - that is, a low enough speed that still allows the wings to generate the necessary support. How do you do that? The memory speed is a property of the system as a whole: it emerges from the activity of pilots and is not identical to the memory of the pilots [3].

The speed markers (representing speed) are just one of many devices that participate in the functional system that gives an account of memory tasks. It is important to note that the properties of functional systems that are mediated by external representations (such as markers of speed) are different from those that use only internal representations, they may, therefore, depend on physical properties of external representational. Factors such as representation and persistence of the sensory modality through which it is accessed can influence the properties of the cognitive system.

The theoretical context from which we study, for example, memory speeds during landing, take complex cooperative systems, not individual minds, as the primitive unit of analysis. And the theory is constructed is explicitly cognitive in that it refers to how information is represented, transformed and transmitted through the system. In the cockpit, the representation of information is constructed in a manner at once distributed among several devices some internal and others external to the crew to them), negotiated between the various participants. You can not say that the meaning of the various representations of the distributed system is specified in advance. What distinguishes, then, this conception of distributed cognition is the adherence to two principles.

- The first postulate is not always the individual, or the processes internal to it, is the correct unit of analysis. An overview of distributed cognition looks for cognitive processes wherever they occur, singling them only based on functional relationships of the elements involved in the process. A cognitive process is not just because it has a brain like his support, or because it involves multiple brains. As noted above, systems that can be characterized as sociotechnical like the cockpit of a plane, can be taken as cognitive units

- The second principle concerns the mechanisms that participate in cognitive processes. The classical approaches tend to assume that cognitive events lie in the manipulation of symbols inside staff. 


\section{Conclusions}

The processes of working memory resulting a rich interaction between internal processes (the pilots), the manipulation of objects (markers of speed, for example) and exchange of information between drivers. In addition, the physical constraints of the environment provides more than just help with memory processes, such as in the case of corrections speed by observing device called salmon. Physical constraints reorganize the distributed cognitive system, using different sets of internal and external processes.

The relationship between communication processes and their senses have been studied in two ways:

a) As an internal property of individuals (or organisms), whether defined as innate, or by the accumulation of experience.

b) Like determinations as possibilities or originating from the external environment in which individuals move. The human mind is vulnerable to phenomena which can modify the behavior expected in jobs, breaking the stability of the process "cognitive" collective and providing conditions that can lead to accidents. The "pseudo-invasion" of the task of "another party", when there are abnormalities that require primarily the Human action is not provided. As metaphorized in Figures 5, 6 and 7.

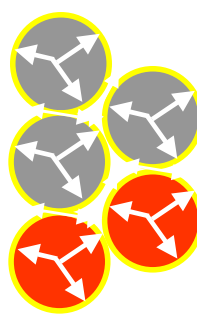

Fig. 5 - Initial: tweaking skills to develop a network of distributed knowledge (neural)

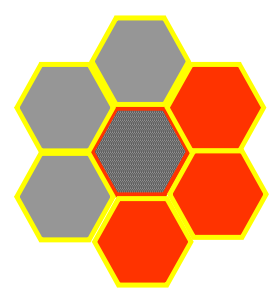

Fig. 6 - Expected result stability of overlapping competences and distributed

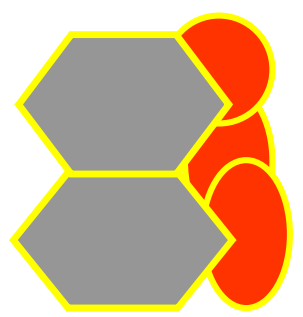

Fig.7 - Deficiencies: deformation in the distribution and execution of actions Asynchrony and instability in humanrobot

Figures. 5, 6 e 7 - The distortion in the distribution of tasks, leading to accidents in the case in aviation HUMAN-COMPUTER

So there are three distinct aspects to consider when studying the distribution of cognitive processes:

- The processes can be distributed among members of a social group;

- The processes may involve coordination between internal and external structures and,

- The processes can be distributed in time, so that the products of earlier events can transform the nature of subsequent events.

What distinguishes, then, this conception of distributed cognition is the adherence to two principles.

- The first postulate is not always the individual, or the processes internal to it, is the correct unit of analysis. An overview of distributed cognition looks for cognitive processes wherever they occur, singling them only based on functional relationships of the elements involved in the process. A cognitive process is not just because it has a brain like his support, or because it involves multiple brains. As noted above, systems that can be characterized as sociotechnical like the cockpit of a plane, can be taken as cognitive units. 
- The second principle concerns the mechanisms that participate in cognitive processes. The classical approaches tend to assume that cognitive events lie in the manipulation of symbols inside staff.

The mixed use of neural networks reveals a unique way of addressing the relationship between communication and senses, which can represent an opportunity to overcome the apparent dichotomy of classical solutions. The possibility of studying these issues through experiments in robotics, computational modeling and ethnography of cognitive systems allows instantiating systems composed of autonomous agents who need to create and negotiate ways to collectively perform tasks. In these systems can then study the precise way in which co-determination of internal and external communications are established.

\section{References}

[1] Port, R.F. \& Van Gelder, T. Mind as motion: explorations in the dynamics of cognition. Cambridge, MA: The MIT Press. 1995.

[2] Elman et al. (1996), Bechtel and Abrahamsen (2002), Artificial Life and approaches based on dynamical systems (Portland publish,)

[3] Hollan, J. D., Hutchins, E. and Kirsh, D. Distributed Cognition: A New Foundation for Human-Computer Interaction Research - Computer Interaction in the New Millennium, 1999 